\title{
Symmetry-Breaking for Solutions of Semilinear Elliptic Equations with General Boundary Conditions *
}

\author{
Joel A. Smoller and Arthur G. Wasserman
}

Department of Mathematics, University of Michigan, Ann Arbor, MI 48109-1003, USA

\begin{abstract}
We study the bifurcation of radially symmetric solutions of $\Delta u+f(u)=0$ on $n$-balls, into asymmetric ones. We show that if $u$ satisfies homogeneous Neumann boundary conditions, the asymmetric components in the kernel of the linearized operators can have arbitrarily high dimension. For general boundary conditions, we prove some theorems which give bounds on the dimensions of the set of asymmetric solutions, and on the structure of the kernels of the linearized operators.
\end{abstract}

\section{Introduction}

We are interested in the bifurcation of radially symmetric solutions of semilinear elliptic equations

$$
\Delta u(x)+f(u(x))=0, \quad x \in B^{n}=\text { an } n \text {-ball, }
$$

into asymmetric ones; when this happens we say that the symmetry-breaks. In a recent paper, [7], we studied this problem for solutions which satisfied (homogeneous) Dirichlet boundary conditions. Our goal here is to consider more general boundary conditions, and to demonstrate some interesting differences which can occur for other boundary conditions. For example, we show that for (homogeneous) Neumann boundary conditions, the symmetry can break in such a way that the asymmetric components in the kernel of the associated linearized operators have arbitrarily high dimensions. In fact, we shall construct a function $f(u)$ and a sequence of monotone radial solutions $\left\{u_{k}\right\}$, such that the asymmetric component of the kernel of the linearized operator about $u_{k}$ has dimension $\left(\begin{array}{c}n+k-2 \\ k\end{array}\right)\left(\frac{n+2 k-2}{n+k-2}\right)$. Thus the symmetry breaks infinitesimally in an increasingly more complicated manner. It should be contrasted with the case of Dirichlet boundary conditions, where for monotone radial solutions, the symmetry can

* Research supported in part by the NSF under Grant No. MCS-8002337 
break only in a most rigid way; namely the asymmetric component of the kernel always has the same fixed dimension $n$ (see [7]). In this case $f(0)<0$ is a necessary condition for the symmetry to break on positive solutions, and when the symmetry breaks on such a solution, it only breaks in the first mode. By contrast, for Neumann boundary conditions, there is no requirement on $\operatorname{sgn} f(0)$, and there can never be symmetry-breaking in the first mode. Indeed there can be, (and is) symmetry breaking in all sufficiently high modes, as shown by our example.

For general homogeneous linear boundary conditions, we prove some theorems which give bounds on the dimensions of the manifolds of asymmetric solutions, and on the structure of the elements in the kernels of the associated linearized operators. Thus, if the symmetry breaks on a monotone radial solution ${ }^{1}$, then we prove that the asymmetric component of this kernel must be irreducible ${ }^{2}$. This fact is sometimes needed to apply certain bifurcation theorems, e.g. [10]. We also show that for any element in this kernel, its radial part cannot have more zeros than the derivative of the radial solution itself. We use this result to estimate the number of irreducible components in the kernel, and hence to bound the number of distinct sets of asymmetric solutions which can bifurcate out of a radial solution.

Certain of our results are extensions of those in [7], where we studied related questions for monotone solutions of the Dirichlet problem. The fairly difficult construction given here, (in Sect. 4), of the existence of an asymmetric element in the kernel of the associated linearized operator, (with homogeneous Neumann boundary conditions), relies on the fact that this operator is self-adjoint with respect to a weighted $L_{2}$-inner product ${ }^{3}$. This allows us to view the problem as a variational one. The success of this approach in turn, is based upon rather careful estimates of the "time-maps" (see $[5,6]$ ), together with an existence theorem proved in [8].

We point out that since radial solutions of our equation satisfy an ordinary differential equation, it is natural to allow the radii of the domains to vary, and to consider orbits of the associated first-order system of equations which satisfy $u(0)=p>0$, and $u^{\prime}(0)=0$. In this context, then, we can take $p$ to be the bifurcation parameter. This is a slightly different approach from the usual one, where one considers the equation $\Delta u+\lambda f(u)=0$, on a fixed domain $\Omega$, and one takes $\lambda$ to be the bifurcation parameter. We could just as well study the equation $\Delta u+f(u)=0$ on the domain $\sqrt{\lambda} \Omega$, i.e., changing the domain by a similarity transformation is the same as changing $\lambda$. We find it more convenient to use $p$ as the bifurcation parameter.

\section{The Equations}

Let $D_{R}^{n}$ be an $n$-ball of radius $R$, centered at the origin with boundary $\partial D_{R^{n}}^{n}$ We consider the equation

$$
\Delta u(x)+f(u(x))=0, \quad x \in D_{R}^{n},
$$

\footnotetext{
${ }^{1}$ So in particular, if it breaks on a positive solution of the Dirichlet problem; see Gidas et al. [3]

${ }^{2}$ That is, under the action of the orthogonal group $O(n)$, it has no proper invariant subspace

${ }^{3}$ We are indebted to Charles Conley for bringing this fact to our attention
} 
together with general linear homogeneous boundary conditions

$$
\alpha u(x)+\beta d u(x) / d n=0, \quad x \in \partial D_{R}^{n} .
$$

Here $\alpha$ and $\beta$ are constants, $\alpha^{2}+\beta^{2}=1$, and $d / d n$ denotes differentiation in the radial direction on $\partial D_{R}^{n}$. The special class of solutions of (2.1), (2.2), depending only on the radius $r=|x|$, and which we term radial solutions, (or sometimes, invariant solutions), satisfy the ordinary differential equation

$$
u^{\prime \prime}(r)+\frac{n-1}{r} u^{\prime}(r)+f(u(r))=0, \quad 0<r<R,
$$

together with the boundary conditions ${ }^{4}$

$$
u^{\prime}(0)=0, \quad \alpha u(R)+\beta u^{\prime}(R)=0 .
$$

We observe that there is exactly one solution $u$ of $(2.3)$ having $u^{\prime}(0)=0$, and $u(0)=p$; we write this solution as $u(r, p)$, and in these terms, we have

$$
u(0, p)=p \text {. }
$$

Throughout this paper we will consider $p$ as the "bifurcation parameter," and prime, ('), will always denote differentiation with respect to the variable $r$. Let

$$
\begin{aligned}
A=\left\{p: \exists T=T_{\alpha}(p): u(\cdot, p) \text { solves }(2.3) u^{\prime}(0)=0,\right. \\
\\
\left.\quad \text { and } \alpha u(T, p)+\beta u^{\prime}(T, p)=0\right\} .
\end{aligned}
$$

Notice that for each $p, T_{\alpha}(p)$ is (at most) a countable set: $T_{\alpha}^{0}(p), T_{\alpha}^{1}(p), \ldots$, where $T_{\alpha}^{M}(p)$ is the "time" that it takes the solution to make $M / 2$ rotations about the line $\alpha u+\beta u^{\prime}=0, M=0,1,2, \ldots$, in the $\left(u, u^{\prime}\right)$-plane.

The function $p \rightarrow T_{\alpha}^{M}(p)$ will be called the $(\alpha, M)$-time map. We will use the notation

$$
T_{D}=T_{1}^{0}, \quad T_{N}=T_{o}^{0}, \quad T_{\alpha}=T_{\alpha}^{0}
$$

to denote the $M=0$ Dirichlet-time map, the $M=0$ Neumann-time map and the general $M=0$ time map, respectively. In this paper we shall be concerned only with these latter functions.

If $u(\cdot, p)$ solves (2.3), (2.4), then the linearized operator $L_{p}: C_{0}^{2}(|x| \leqq R) \rightarrow C(|x|$ $\leqq R),\left(R=T_{\alpha}(p)\right)$, defined on the class of $C^{2}$ functions on $|x| \leqq R$ which satisfy the boundary conditions (2.2), into the continuous functions on the same domain, is defined by

$$
L_{p} v(x)=\Delta v(x)+f^{\prime}(u(|x|, p)) v(x), \quad|x|<R .
$$

We say that $v \neq 0$ is in the kernel of $L_{p}$, if $v$ satisfies the linearized equation

$$
L_{p} v(x)=0, \quad|x|<R,
$$

together with the boundary conditions

$$
\alpha v(x)+\beta d v(x) / d n=0, \quad|x|=R .
$$

\footnotetext{
${ }^{4}$ The condition $u^{\prime}(0)=0$ is necessary if $u$ is to be differentiable at the origin
} 
That is, $v$ is an eigenvector of $L_{p}$ corresponding to the eigenvalue zero. Using the implicit function theorem, we see that if $u(\cdot, p)$ is a "bifurcation point," then zero is an eigenvalue of $L_{p}$.

We shall now briefly review some facts concerning the eigenfunctions of the Laplacian $\Delta$ on $S^{n-1}$, and the associated spherical harmonic decomposition of functions defined on an $n$-ball; for more details, see, e.g., $[1,4]$.

The eigenvalues of $\Delta$ on $S^{n-1}$ are given by

$$
\lambda_{N}=-N(N+n-2), \quad N=0,1,2, \ldots .
$$

Let $\left\{\Phi_{i}^{N}: 1 \leqq \mathrm{i} \leqq \mathrm{k}_{N}\right\}$ denote a basis for the $N^{\text {th }}$ eigenspace of $\Delta$; i.e., for the eigenfunctions of $\Delta$ on $S^{n-1}$, with eigenvalue $\lambda_{N}$. Then we have the following facts:

(i) The dimension of the $N^{\text {th }}$ eigenspace is

$$
L_{N}=\left(\begin{array}{c}
n+N-2 \\
N
\end{array}\right)\left(\frac{n+2 N-2}{n+N-2}\right), \quad N=0,1,2, \ldots
$$

(ii) The set $\left\{\Phi_{i}^{N}: 1 \leqq i \leqq k_{N}, i=0,1,2, \ldots\right\}$ forms a complete orthonormal set on $L^{2}\left(S^{n-1}\right)$.

As a consequence of these facts, any function $h$ on $S^{n-1}$ can be written as

$$
h=\sum_{i, N}\left\langle h, \Phi_{i}^{N}\right\rangle \Phi_{i}^{N},
$$

where

$$
\left\langle h, \Phi_{i}^{N}\right\rangle=\int_{S^{n-1}} h(\theta) \Phi_{i}^{N}(\theta) d \theta .
$$

Thus, for any function $v(r, \theta)$ on an $n$-ball $|x| \leqq R$, we may write $v$ in the form

$$
v(r, \theta)=\sum_{i, N}\left\langle v(r, \cdot), \Phi_{i}^{N}(\cdot)\right\rangle \Phi_{i}^{N}(\theta)=\sum_{i, N} a_{i, N}(r) \Phi_{i}^{N}(\theta),
$$

for each fixed $r, 0 \leqq r \leqq R$. Now suppose in particular, that $v$ satisfies (2.8), and (2.9). Then using (2.12), we find

$$
\sum_{i, N}\left[a_{i, N}^{\prime \prime}+\frac{n-1}{r} a_{i, N}^{\prime}+\left(\frac{\lambda_{N}}{r^{2}}+f^{\prime}(u(r, p))\right) a_{i, N}\right] \Phi_{i}^{N}=0 .
$$

It follows that all the coefficients in the above sum must be zero. Thus we see that:

1) Each $a_{i, N}$ satisfies a second-order ordinary differential equation;

2) For a fixed $N$, each $a_{i, N}$ satisfies the same ordinary differential equation, $P_{N}\left(a_{i, N}\right)=0$;

3) For each $N>0$, there is only one solution (up to a constant multiple) to the equation $P_{N}(a)=0$, which is finite at $r=0$.

This last fact holds since the Wronskian of any two solutions satisfies the firstorder equation $w^{\prime}+(n-1) w / r=0$, and hence $w$ either "blows-up" at $r=0$, or $w \equiv 0$.

Now in view of these facts, we see that we can write each $a_{i, N}(r)$ in the form $a_{i, N}(r)=c_{i}^{N} a_{N}(r)$, and hence (2.12) becomes

$$
v(r, \theta)=\sum_{N} \sum_{i} a_{i, N}(r) \Phi_{i}^{N}(\theta)=\sum_{N} \sum_{i} c_{i}^{N} \Phi_{i}^{N}(\theta) a_{N}(r)=\sum_{N} a_{N}(r) \tilde{\Phi}_{N}(\theta),
$$

where

$$
\tilde{\Phi}_{N}(\theta)=\sum_{i} c_{i}^{N} \Phi_{i}^{N}(\theta)
$$


and $a_{N}$ satisfies the ordinary differential equation

$$
a_{N}^{\prime \prime}(r)+\frac{n-1}{r} a_{N}^{\prime}(r)+\left(f^{\prime}(u(r, p))+\frac{\lambda_{N}}{r^{2}}\right) a_{N}(r)=0,
$$

on $0<r<R$. Moreover, if $v$ is a solution of $(2.8),(2.9)$, then since

$$
a_{N}(r)=\int_{S^{n-1}} v(r, \theta) \tilde{\Phi}_{N}(\theta) d \theta,
$$

we find that for $N>0$ (cf. [7]), $a_{N}$ satisfies the boundary conditions

$$
a_{N}(0)=0, \quad \alpha a_{N}(R)+\beta a_{N}^{\prime}(R)=0, \quad N \geqq 1 .
$$

Also, (2.13) and (2.10) show that $a_{0}$ satisfies the boundary conditions

$$
a_{0}^{\prime}(0)=0, \quad \alpha a_{0}(R)+\beta a_{0}^{\prime}(R)=0 .
$$

If $u(\cdot, p)$ is a radial solution of $(2.1),(2.2)$ and an asymmetric solution bifurcates out of $u(\cdot, p)$, then it is not hard to show that the kernel of $L_{p}$ must contain a nontrivial asymmetric element (see, e.g., [2]). Accordingly, we make the following definition (see [7]).

Definition 2.1. If $u(\cdot, p)$ is a radial solution of $(2.1),(2.2)$, then we say that

(i) The symmetry breaks infinitesimally on $u(\cdot, p)$ if there exists an element in the kernel of the linearized operator $L_{p}$, which is asymmetric; i.e., non-radial.

(ii) The symmetry breaks on $u(\cdot, p)$, if an asymmetric solution bifurcates out of $u(\cdot, p)$.

In these terms as we have remarked above, symmetry-breaking implies infinitesimal symmetry breaking. Note too that if the kernel of $L_{p}$ contains a noninvariant element, then from (2.12) and the fact that $u(\cdot, p)$ is invariant, we can find an element in the kernel which is of the form $a_{N}(r) \widetilde{\Phi}_{N}(\theta)$.

The above considerations show that the kernel of $L_{p}$ is different from zero if and only if there is a non-zero solution of (2.13) and (2.14) or (2.15). Evidently if the problem (2.13), (2.14) has a non-zero solution, then the symmetry breaks infinitesimally at $u(\cdot, p)$. In order to show that the symmetry actually breaks on $u(\cdot, p)$, we shall appeal to the Crandell-Rabinowitz bifurcation theorem $[9$, p. 173]:

Theorem 2.2. Let $U=S \times V$ be an open subset of $\mathbb{R} \times X$, and let $F \in C^{2}(U, Y)$, where $X$ and $Y$ are Banach spaces. Suppose that $F(\lambda, 0)=0$ for all $\lambda \in S$, and let $L_{0}=D_{2} F\left(\lambda_{0}, 0\right), L_{1}=D_{1} D_{2} F\left(\lambda_{0}, 0\right)$. Assume that the following conditions hold:

(a) The null space of $L_{0}$ is one-dimensional, spanned by $u_{0}$.

(b) The range of $L_{0}$ has co-dimension 1.

(c) $L_{1} u_{0}$ is not in the range of $L_{0}$.

Let $Z$ be any closed subspace of $X$ such that $X=\left[\operatorname{span} u_{0}\right] \oplus Z$. Then there is a $\delta>0$, and a $C^{1}$-curve $(\lambda, \phi):(-\delta, \delta) \rightarrow \mathbb{R} \times Z$ such that: (i) $\lambda(0)=\lambda_{0}$; (ii) $\phi(0)=0$; and $F\left(\lambda(s), s\left(u_{0}+\phi(s)\right)\right) \equiv 0$ for $|s|<\delta$.

This completes the background material which we shall need in the subsequent sections. 


\section{General Boundary Conditions}

We begin this section with a simple comparison result concerning solutions $a$ of the equation

$$
r^{2}\left\{a^{\prime \prime}(r)+\frac{\lambda}{r} a^{\prime}(r)\right\}+\phi(r) a(r)=0, \quad 0<r<R,
$$

$(\lambda=$ constant $)$, which is independent of any boundary conditions that a satisfies. This then will be applied to Eqs. (2.3), (2.4), to yield some general theorems concerning the functions $a_{N}(r)$. Afterwards, we shall prove some results which apply to particular boundary conditions. Here is the useful comparison theorem, which is really a slight variant of the well-known Sturm comparison theorem.

Proposition 3.1. Suppose that $b(r)$ is a solution of the equation

$$
r^{2}\left\{b^{\prime \prime}(r)+\frac{\lambda}{r} b^{\prime}(r)\right\}+\theta(r) b(r)=0, \quad 0<r<R,
$$

and $a(r)$ solves (3.1). Assume that $\phi$ and $\theta$ are continuous on $0 \leqq r \leqq R$, and $\theta(r) \geqq \phi(r)$ on this range. If $a \neq 0$, then $b$ has a zero between any two adjacent zeros of $a$.

Proof. If $a(r)=a^{\prime}(r)=0$ for some $r, 0<r \leqq R$, then $a \equiv 0$. Thus the zeros of $a$ can only accumulate at $r=0$. Let $r_{1}$ and $r_{2}$ be adjacent zeros of $a, 0 \leqq \mathrm{r}_{1}<r_{2}$, where $a(r)>0$ if $r_{1}<r<r_{2}$.

Now multiply (3.1) by $r^{\lambda-2} b(r),(3.2)$ by $r^{\lambda-2} a(r)$, subtract, and integrate from $r_{1}$ to $r_{2}$. This gives

$$
r^{\lambda}\left[a^{\prime}(r) b(r)-a(r) b^{\prime}(r)\right]_{r_{1}}^{r_{2}}+\int_{r_{1}}^{r_{2}}(\phi(r)-\theta(r)) a(r) b(r) r^{\lambda-2} d r=0,
$$

so that

$$
r_{2}^{\lambda} a^{\prime}\left(r_{2}\right) b\left(r_{2}\right)-r_{1}^{\lambda} a^{\prime}\left(r_{1}\right) b\left(r_{1}\right)+\int_{r_{1}}^{r_{2}}(\phi-\theta) a b r^{\lambda-2} d r=0 .
$$

If $b$ had no zero in the interval $r_{1}<r<r_{2}$, then $b$ would be of one sign in this interval, thus violating (3.3)

We shall now obtain some consequences of this proposition.

Corollary 3.2. Let $a_{M}$ and $a_{\mathrm{K}}$ be non-zero solutions of the equations

$$
\begin{gathered}
a_{M}^{\prime \prime}+\frac{n-1}{r} a_{M}^{\prime}+\left(f^{\prime}(u)+\frac{\lambda_{M}}{r^{2}}\right) a_{M}=0, \\
a_{K}^{\prime \prime}+\frac{n-1}{r} a_{K}^{\prime}+\left(f^{\prime}(u)+\frac{\lambda_{K}}{r^{2}}\right) a_{K}=0
\end{gathered}
$$

on an interval $0<r<R$, where the $\lambda_{N}$ 's are defined in (2.10). If $K>M$, then $a_{M}$ has a zero between any two adjacent zeros of $a_{K}$.

Proof. Using (2.10), we see that $\lambda_{M}>\lambda_{K}$. Thus we can multiply each of the differential equations by $r^{2}$, and then apply the propostion. 
Suppose now that $u$ is a non-constant radial solution of (2.1) on the $n$-ball $|x|<R$. Then $u$ satisfies (2.3), and since $\lambda_{1}=1-n$ we see that $w \equiv u^{\prime}$ is a solution of the equation

$$
a_{1}^{\prime \prime}(r)+\frac{n-1}{r} a_{1}^{\prime}(r)+\left(f^{\prime}(u(r))+\frac{\lambda_{1}}{r^{2}}\right) a_{1}=0, \quad 0<r<R .
$$

This gives the following two corollaries.

Corollary 3.3. If $N>1$, then on the interval $0 \leqq r \leqq R, u^{\prime}$ must change sign between any two adjacent zeros of $a_{N}$.

We now consider the general case where $u^{\prime}$ is allowed to change sign on an interval. Here is the main result.

Theorem 3.4. Let $u$ be a solution of (2.3). Assume that $u^{\prime}$ has $k$ zeros on the interval $0 \leqq r<R$, and that $a_{N} \neq 0$ solves (2.13), where $N \geqq 1$. Then $a_{N}$ has at most $k$ zeros on this interval. If in addition $u$ satisfies the boundary conditions (2.4), then there are at most $k$ positive integers $N_{1}, N_{2}, \ldots, N_{k}$, for which $a_{N_{j}}$ is a (non-trivial) solution of (2.13), (2.14), $1 \leqq j \leqq k$.

Before giving the proof of this theorem, some remarks are in order. First, if we consider positive solutions of (2.1), together with Dirichlet boundary conditions $[\alpha=1, \beta=0$ in (2.4)], then Gidas et al. [3], have shown that $u$ must be a monotone decreasing radial function. In this case $k=1,(N=1)$, and $a_{1}$ has exactly one zero on $0<r \leqq R$; this is in agreement with the result found in [7]. Next, if we consider monotone solutions of (2.3) together with homogeneous Neumann boundary conditions $\left[\alpha=0, \beta=1\right.$ in (2.4)], then here too $k=1$, and so again $a_{N}$ has exactly one zero. Finally, note that if $k>1$, and there are two non-zero functions, say $a_{M} \neq 0$, and $a_{K} \neq 0,(K \neq M)$, which are solutions of (3.4) and (3.5), for the same $u=u(\cdot, p)$, then the kernel of the linearized operator $L_{p}[\operatorname{see}(2.7)]$ contains the two non-zero elements $a_{K}(r) \tilde{\Phi}_{K}(\theta)$, and $a_{M}(r) \tilde{\Phi}_{M}(\theta)$. Since $K \neq M$, the subspaces generated by these two elements are distinct, proper, and both are invariant under the orthogonal group. Thus the asymmetric component of the kernel of $L_{p}$ cannot be irreducible. It follows that the bifurcation theorem discussed in Sect. 2 does not apply since hypothesis $\left(a_{2}\right)$ is not satisfied. (But some statements concerning the bifurcation of solutions can be made; these will be considered in a future publication.)

On the other hand, the problem of demonstrating that there can be two nonzero functions $a_{K}$ and $a_{M}$, as above, is quite delicate, and is still unresolved.

Proof of Theorem 3.4. Suppose that $a_{N}$ has $(k+1)$-zeros on the interval;i.e., $a_{N}\left(z_{0}\right)$ $=a_{N}\left(z_{1}\right)=\ldots=a_{N}\left(z_{k}\right)=0$, where $0=z_{0}<z_{1}<\ldots<z_{k}<R$. From Corollary $3.3, u^{\prime}$ has a zero on each interval $\left(z_{i-1}, z_{i}\right), i=1,2, \ldots, k$. But as $u^{\prime}\left(z_{0}\right)=0$, we see that $u^{\prime}$ has $(k+1)$-zeros on $[0, R)$. This is a contradiction; thus $a_{N}$ can have at most $k$-zeros. In order to prove the last assertion, we need the following lemma, which is of some interest by itself.

Lemma 3.5. Under the hypotheses of the theorem, (including the fact that u satisfies (2.4)), suppose that $K$ and $M$ are positive integers, $K>M$, and that $a_{M}$ and $a_{K}$ are 
non-zero solutions of (3.5) and (3.6), respectively, together with the boundary conditions (2.4). Then on the interval $0 \leqq r \leqq R$, the following inequalities hold:

$$
\text { \#zeros of } a_{K}<\# \text { zeros of } a_{M} \leqq \# \text { zeros of } u^{\prime} \text {. }
$$

Proof. The second inequality is a consequence of Corollary 3.3. In order to prove the first, we note that $\lambda_{M}>\lambda_{K}$ [see (2.10)]; thus from Corollary 3.2, \# zeros of $a_{K}$ $\leqq \#$ of zeros of $a_{M}$. If equality holds, then we have

$$
\begin{gathered}
a_{M}(0)=a_{M}\left(z_{1}\right)=\ldots=a_{M}\left(z_{s}\right)=0, \\
a_{K}(0)=a_{K}\left(w_{1}\right)=\ldots=a_{K}\left(w_{s}\right)=0, \quad s \leqq k,
\end{gathered}
$$

where $0<z_{1}<\ldots<z_{s}$ and $0<w_{1}<\ldots<w_{s}$, and these are all of the zeros of $a_{M}$ and $a_{K}$, respectively. Using Corollary 3.2 again, we get the inequalities

$$
0<z_{1}<w_{1}, \quad w_{i}<z_{i+1}<w_{i+1}, \quad i=1,2, \ldots, s-1 .
$$

There is no loss in generality to assume that $a_{M}>0$ and $a_{K}>0$ on $\left(0, z_{1}\right)$. Then the product $a_{M} a_{K}$ is negative on the intervals $\left(z_{i}, w_{i}\right), 1 \leqq i \leqq s$, and positive on the intervals $\left(w_{i}, z_{i+1}\right), 1 \leqq i \leqq s-1$. We distinguish two cases; namely $w_{s}<R$, and $w_{\mathrm{s}}=R$.

Suppose that $w_{s}<R$; then $a_{M} a_{K}>0$ on $\left(w_{s}, R\right)$. Now if we multiply (3.4) by $r^{n-1} a_{K}$, and (3.5) by $r^{n-1} a_{M}$, subtract, and integrate from $w_{s}$ to $R$, we get

$$
\left.r^{n-1}\left(a_{K}^{\prime} a_{M}-a_{K} a_{M}^{\prime}\right)\right|_{w_{s}} ^{R}+\int_{w_{s}}^{R}\left(\lambda_{k}-\lambda_{M}\right) a_{K}(r) a_{M}(r) r^{n-3} d r=0 .
$$

Now since $\alpha a_{K}(R)+\beta a_{K}^{\prime}(R)=0=\alpha a_{M}(R)+\beta a_{M}^{\prime}(R)$, we see that $\left(a_{K} a_{M}^{\prime}\right.$ $\left.-a_{K}^{\prime} a_{M}\right)(R)=0$. Thus $(3.6)$ reduces to

$$
-w_{s}^{n-1} a_{K}^{\prime}\left(w_{s}\right) a_{M}\left(w_{s}\right)+\int_{w_{s}}^{R}\left(\lambda_{K}-\lambda_{M}\right) a_{K}(r) a_{M}(r) r^{n-1} d r=0 .
$$

However, this is impossible since both terms on the left-hand side of (3.7) are negative.

We now consider the case where $w_{s}=R$. Then from (2.14),

$$
0=\alpha a_{K}(R)+\beta a_{K}^{\prime}(R)=\beta a_{K}^{\prime}(R) .
$$

Since $a_{K} \neq 0$, and $a_{K}(R)=0$, it follows that $a_{K}^{\prime}(R) \neq 0$, and so $\beta=0$. Therefore,

$$
0=\alpha a_{M}(R)+\beta a_{M}^{\prime}(R)=\alpha a_{M}(R)=\alpha a_{M}\left(w_{s}\right),
$$

so that $a_{M}\left(w_{s}\right)=0$. Thus $a_{M}$ has $s+2$ zeros; this is again a contradiction, and the proof of the lemma is complete.

We can now easily complete the proof of Theorem 3.4. For suppose that there is a positive integer $N_{1}$ such that $a_{N_{1}}$ has $k$ zeros on $[0, R)$. Then our lemma implies that there can be at most $(k-1)$-indices $N_{2}, \ldots, N_{k}$ for which $a_{N_{i}} \neq 0,2 \leqq i \leqq k$.

Corollary 3.6. If $u^{\prime}$ is a solution of (2.3), (2.4), and is of constant sign on an interval $0<r<R$, then there can be at most one $N>1$ for which $a_{N} \neq 0$. Moreover for this $N$, $a_{N}(r) \neq 0$ on $0<r<R$. 
This last result indicates that if $u^{\prime}$ is of constant sign on an interval $0<r<R$, then the unique non-zero $a_{N}$, (if it exists!), could conceivably be shown to exist via a variational approach, as the principal eigenfunction of some operator (see $[9$, Chap. 11]). In Sect. 4, we shall show that this program can indeed be carried out in the case that $u$ satisfies homogeneous Neumann boundary conditions $[\alpha=0, \beta=1$ in (2.4)].

As a final result along these lines, we have the following corollary to Lemma 3.5.

Corollary 3.7. Suppose that $a_{M}$ and $a_{K}$ are non-zero solutions of (3.4) and (3.5), respectively, and both satisfy the boundary conditions (2.4). If $M \neq K$, then $a_{M}$ and $a_{K}$ cannot both be of constant sign on $0<r<R$.

We turn now to the problem of radial bifurcation; that is, we shall study the question of when $a_{0}(r) \neq 0$. Thus using (2.13) and (2.10), we see that $a_{0}$ satisfies the equation

$$
a_{0}^{\prime \prime}(r)+\frac{n-1}{r} a_{0}^{\prime}(r)+f^{\prime}(u(r, \bar{p})) a_{0}(r)=0, \quad 0<r<R,
$$

together with the boundary conditions (2.15). Of course, we assume here that we are given a particular radial solution $u=u(r, \bar{p})$ of $(2.3),(2.4)$ on $0<r<R$, where $R=T_{\alpha}(\bar{p})$, [cf. $\left.(2.5 \mathrm{ff})\right]$.

If $a_{0} \neq 0$ solves (3.8), (2.15), then we say that $u(\cdot, \bar{p})$ is radially degenerate; otherwise $u(\cdot, \bar{p})$ is called radially non-degenerate. Here is the theorem which characterizes infinitesimal radial bifurcation.

Theorem 3.8. Suppose that $\mathscr{U}=\left\{u(\cdot, p): p_{1}<p<p_{2}\right\}$ is a family of solutions of (3.8), (2.15). Then $u(\cdot, \vec{p}) \in \mathscr{U}$ is radially degenerate if and only if ${ }^{5}$

$$
\left\{\alpha u^{\prime}\left(T_{\alpha}(\bar{p}), \bar{p}\right)+\beta u^{\prime \prime}\left(T_{\alpha}(\bar{p}), \bar{p}\right)\right\} T_{\alpha}^{\prime}(\bar{p})=0 .
$$

Remark. We note that this extends a result in [6] stated for Dirichlet boundary conditions, $(\alpha=1, \beta=0)$. Also observe that for Neumann boundary conditions $(\alpha=0, \beta=1),(3.9)$ reduces to $-f\left(u\left(T_{N}(\bar{p}), \bar{p}\right)\right) T_{N}^{\prime}(\bar{p})=0$, and as $f\left(u\left(T_{N}(\bar{p}), \bar{p}\right)\right) \neq 0$, (if we consider non-constant solutions), this means $T_{N}^{\prime}(\bar{p})=0$.

Proof. We differentiate ${ }^{6}(2.3)$ with respect to $p$ to get

$$
u_{p}^{\prime \prime}+\frac{n-1}{r} u_{p}^{\prime}+f^{\prime}(u) u_{p}=0,
$$

and thus $u_{p}(\cdot, \bar{p})$ satisfies the linearized equation (3.8). Moreover since $u^{\prime}(0, p)=0$ for all $p, p_{1}<p<p_{2}$, we have $u_{p}^{\prime}(0, \bar{p})=0$, so that $u_{p}(\cdot, \tilde{p})$ satisfies the first boundary condition in (2.15). To obtain the second boundary condition, we recall that by definition of $T_{\alpha}(p)$, we have

$$
\alpha u\left(T_{\alpha}(p), p\right)+\beta u^{\prime}\left(T_{\alpha}(p), p\right)=0, \quad p_{1}<p<p_{2} .
$$

\footnotetext{
${ }^{5}$ In [6] it is shown that $T_{a}$ is a $C^{1}$ function if $f$ is contimuous

${ }^{6}$ In [6] it is shown that $u, u^{\prime}, u^{n}$ are $C^{1}$ functions of $p$ if $f$ is continuous
} 
Then differentiating with respect to $p$ and evaluating at $\bar{p}$ gives

$$
\left\{\alpha u^{\prime}\left(T_{\alpha}(\bar{p}), \bar{p}\right)+\beta u^{\prime \prime}\left(T_{\alpha}(\bar{p}), \bar{p}\right)\right\} T_{\alpha}^{\prime}(\bar{p})+\alpha u_{p}\left(T_{\alpha}(\bar{p}), \bar{p}\right)+\beta u_{p}^{\prime}\left(T_{\alpha}(\bar{p}), \bar{p}\right)=0 .
$$

Thus, if (3.9) holds, then $u_{p}(\cdot, \bar{p})$ satisfies the boundary conditions (2.15), and since $u_{p}(0, \bar{p})=1$ [Cf. (2.5)], we see that $u_{p}(\cdot, \bar{p})$ is a non-trivial solution of $(3.8),(2.15)$, so that $u(\cdot, \bar{p})$ is radially degenerate. Conversely, if $u(\cdot, \bar{p})$ is radially degenerate, let $z$ be a non-trivial solution of (3.8), (2.15). Then $z^{\prime}(0)=0, z \neq 0$, so $z(0) \neq 0$. Thus $u_{p}(r, \bar{p})$ $=z(r) / z(0)$, since they both satisfy $(2.13), N=0$, and the same initial conditions. Hence (3.9) follows from (3.10).

We remark that this theorem holds for the general time maps $T_{\alpha}^{M}$ where in (3.9) $T_{\alpha}$ is replaced by $T_{\alpha}^{M}$; the proof is the same as the one given here.

We now give an extension of a result in [6], to more general boundary conditions.

Theorem 3.9. Let $u(\cdot, p)$ be a non-constant solution of (2.3) which satisfies the boundary conditions

$$
\alpha u(R, p)+\beta u^{\prime}(R, p)=c, \quad\left(R=T_{\alpha}(p)\right) .
$$

Then referring to solutions $a_{N}$ of (2.13), (2.14), the following hold:

(i) There is a solution $a_{1} \neq 0$ if and only if

$$
\alpha u^{\prime}(R, p)+\beta u^{\prime \prime}(R, p)=0 .
$$

(ii) If (3.12) holds, and $u^{\prime} \leqq 0$ on $0 \leqq r \leqq R$, then $a_{N} \equiv 0$ if $N>1$.

Remarks. If we consider homogeneous Dirichlet boundary conditions $(\alpha=1, \beta=0$, $c=0),(3.12)$ reduces to $u^{\prime}(R, p)=0$, a result obtained in [6]. In our theorem, the ray $u=0, u^{\prime} \equiv v \leqq 0$, is replaced by the ray $\alpha u+\beta v=c, v \leqq 0$; see Fig. $1,(c=0)$.

Proof of Theorem 3.9. If (3.12) holds, we set $a_{1}(r)=u^{\prime}(r, p), 0 \leqq r \leqq R$. Then $a_{1}$ satisfies (2.13) for $N=1$, and $a_{1}$ also satisfies the correct boundary conditions (2.14). Furthermore, in this case, $a_{N} \equiv 0$ if $N>1$, in view of Lemma 3.5.

It remains to show that (3.12) holds if $a_{1}$ is a non-trivial solution of (2.13) (for $N=1$ ), and (2.14). Now from the equation

$$
R^{n-1}\left(a_{1} w^{\prime}-a_{1}^{\prime} w\right)(R)+\int_{0}^{R} r^{n-3}\left(\lambda_{1}+(n-1)\right) a_{1}(r) w(r) d r=0,
$$

(with, $w=u^{\prime}$, as usual), we have, since $\lambda_{1}+n-1=0$,

$$
w^{\prime}(R) a_{1}(R)-w(R) a_{1}^{\prime}(R)=0 .
$$

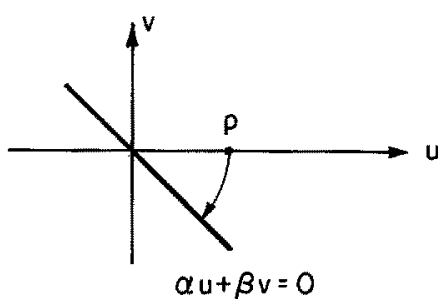

Fig. 1

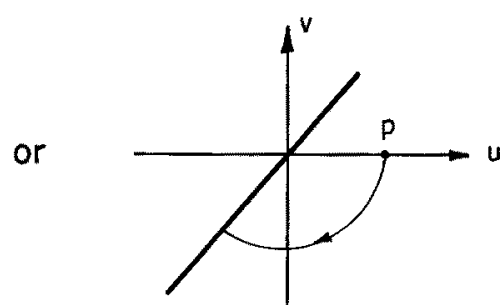

$\alpha u+\beta v=0$ 
But also, $\alpha a_{1}(R)+\beta a_{1}^{\prime}(R)=0$, and since $\left(a_{1}(R), a_{1}^{\prime}(R)\right) \neq(0,0)$, the determinant of this system of linear homogeneous equations must vanish. Therefore $\alpha w(R)$ $+\beta w^{\prime}(R)=0$; thus (3.12) holds.

This last theorem shows that for solutions of (2.3), (2.4), which have $u^{\prime} \leqq 0$, the general homogeneous linear boundary conditions yield results for infinitesimal symmetry-breaking which are exactly the same as those for homogeneous Dirichlet boundary conditions. Notice that if we consider homogeneous Neumann boundary conditions $(\alpha=0, \beta=1)$, then (3.12) implies that $u^{\prime \prime}(R, p)=0$, and since $u^{\prime}(R, p)=0,(2.3)$ shows that $f(u(R, p))=0$. Thus the solution $u(\cdot, p)$ must be the constant function $u(R, p)$. It follows that for non-constant solutions of (2.3), (2.4), with $\alpha=0, \beta=1, a_{1}$ must necessarily be the zero function. We state this formally for later use.

Theorem 3.10. Let $u(\cdot, p)$ be a non-constant monotone solution of $(2.3)$, together with homogeneous Neumann boundary conditions $(2,4)(\alpha=0, \beta=1)$. Then there are no non-trivial solutions $a_{1}$ of (2.13) $(N=1)$ and (2.14) $(\alpha=0, \beta=1)$.

Thus symmetry-breaking for (monotone) solutions of the Neumann problem must necessarily occur in a more complex manner than that for (monotone) radial solutions of the Dirichlet problem. In the next section, we shall make this statement more explicit.

\section{Homogeneous Neumann Boundary Conditions}

We consider monotone radial solutions of (2.3), which satisfy homogeneous Neumann boundary conditions, $u^{\prime}(0)=u^{\prime}(R)=0$. Our goal is to construct an example of symmetry-breaking for the Neumann problem. In this section we shall show that there is infinitesimal symmetry-breaking, and in Sect. 5 we shall show that the symmetry actually breaks.

Using Theorem 3.10, we see that to construct an example of infinitesimal symmetry breaking for the Neumann problem is fairly more difficult than the corresponding construction for the Dirichlet problem. Indeed, in the latter case, $a_{N} \equiv 0$ if $N>1$, and the only possible non-zero mode is $a_{1}$, which turns out to be the function $u^{\prime}$. The question thus arises as to which mode can "survive" for the Neumann problem; i.e., for which $N$, (necessarily $N>1$, by Theorem 3.10), can $a_{N}$ be non-zero? Of course, in view of Corollary 3.6 , for any given monotone radial solution $u(\cdot, p)$, at most one $a_{N}(N>0)$ can be non-zero - but which one? We shall show here that it is possible to have $a_{N} \neq 0$ for arbitrarily high indices $N$. More precisely, we shall construct an example of a function $f$, and a bounded, increasing sequence of points $\left\{p_{k}\right\}$, for which there is a solution $u\left(\cdot, p_{k}\right)$ of $(2.3)$ and $(2.4),(\alpha=0$, $\beta=1$ ), and such that there exists a non-zero solution of each problem,

$$
\begin{gathered}
a^{\prime \prime}(r)+\frac{n-1}{r} a^{\prime}(r)+\left(f^{\prime}\left(u\left(r, p_{k}\right)\right)+\frac{\lambda_{k}}{r^{2}}\right) a(r)=0, \quad 0<r<T_{N}\left(p_{k}\right), \\
a(0)=a^{\prime}\left(T_{N}\left(p_{k}\right)\right)=0 .
\end{gathered}
$$

This will show that the asymmetric component of the kernel of the linearized operator can have arbitrarily high dimension [see (2.11)]. It is worth mentioning 
again that for the Dirichlet problem, the asymmetric component of the corresponding kernel always has dimension $n$. In Sect. 5 we shall show that the symmetry actually breaks on each of the solutions $u\left(\cdot, p_{k}\right)$. We proceed now with the details.

Let $u=u(r, p)$ be a (non-constant) solution of the equation

$$
u^{\prime \prime}(r)+\frac{n-1}{r} u^{\prime}(r)+f(u(r))=0, \quad 0<r<R,
$$

together with the homogeneous Neumann boundary conditions

$$
u(0)=p, \quad u^{\prime}(0)=u^{\prime}(R)=0 .
$$

Here, (by definition)

$$
R=T_{N}(p),
$$

and $u$ is monotone decreasing; i.e.,

$$
u^{\prime}(r, p) \leqq 0, \quad 0 \leqq r \leqq R .
$$

Now in order for the symmetry to break infinitesimally on this solution, it is necessary and sufficient that for some positive integer $k$, there exists a non-trivial solution of the following boundary-value problem:

$$
a_{k}^{\prime \prime}(r)+\frac{n-1}{r} a_{k}^{\prime}(r)+\left(f^{\prime}(u(r, p))+\frac{\lambda_{k}}{r^{2}}\right) a_{k}(r)=0, \quad 0<r<R,
$$

and

$$
a_{k}(0)=a_{k}^{\prime}(R)=0 .
$$

In view of Corollary 3.6, and Theorem $3.10, k>1$, and $a_{k}$ is of constant sign on $0 \leqq r$ $\leqq R$. Moreover, there can be at most one integer $k>1$ for which $a_{k} \neq 0$. This last fact implies that under our hypotheses, the non-symmetric part of the kernel of the linearized operator is irreducible ${ }^{7}$; this result will be used in Sect. 5 when we prove that bifurcation actually occurs.

We now turn our attention to the construction of a non-zero solution of (4.5), (4.6). Thus, let $u(r, p)$ satisfy (4.2)-(4.4), set $R=T_{N}(p)$, and define a function $q_{k}^{p}(r)$ by

$$
q_{k}^{p}(r)=f^{\prime}(u(r, p))+\lambda_{k} / r^{2}, \quad 0<r \leqq R .
$$

Next, we define a space of functions $\Phi_{p}$ by

$$
\Phi_{p}=\left\{\phi \in C^{2}[0, R]: \phi(0)=\phi^{\prime}(R)=0\right\}, \quad R=T_{N}(p) .
$$

Finally, let $L_{k}^{p}$ be the linear operator on $\Phi_{p}$ into $C[0, R]$ given by

$$
L_{k}^{p} \phi=\phi^{\prime \prime}+\frac{n-1}{r} \phi^{\prime}+q_{k}^{p} \phi .
$$

Observe that if we can find a non-zero function $\phi \in \Phi_{p}$ for which $L_{k}^{p} \phi=0$, then this $\phi$ can serve as the desired $a_{k}$; this will demonstrate that the symmetry breaks infinitesimally in the $k^{\text {th }}$ mode, on the radial solution $u(\cdot, p)$.

\footnotetext{
${ }^{7}$ See footnote 2 , and $[4$, p. 417$]$
} 
In order to carry out this program, we shall use a variational approach. Thus, we define an inner product on $\Phi_{p}$ by

$$
\langle\phi, \psi\rangle=\int_{0}^{R} \phi(r) \psi(r) r^{n-1} d r, \quad \phi, \psi \in \Phi_{p} .
$$

We claim that $L_{k}^{p}$ is self-adjoint with respect to this inner product. Thus if $\phi \in \Phi_{p}$, we can write $r^{n-1} L_{k}^{p} \phi=\left(r^{n-1} \phi^{\prime}\right)^{\prime}+q_{k}^{p} \phi r^{n-1}$, so that if also $\psi \in \Phi_{p}$, integrating by parts twice proves the claim. Now it is well-known, see [9, Chap. 11], that the principal eigenvalue, $\mu_{k}^{p}$, of $L_{k}^{p}$ has a variational characterization; namely

$$
\mu_{k}^{p}=\sup \left\{\left\langle L_{k}^{p} \phi, \phi\right\rangle: \phi \in \Phi_{p},\|\phi\|^{2}=1\right\} .
$$

Or, if we integrate by parts, we can write

$$
\mu_{k}^{p}=\sup \int_{0}^{R}\left\{-\left(\phi^{\prime}(r)\right)^{2}+q_{k}^{p}(r) \phi(r)^{2}\right\} r^{n-1} d r, \quad R=T_{N}(p),
$$

where the supremum is taken over the same class of functions as before.

We shall make use of these notions in the following way. Thus we shall construct a function $f(u)$ defined on $\mathbb{R}$, having the following property:

There is an integer $k_{0} \geqq 2$ such that if $k \geqq k_{0}$, there exists a (nontrivial) solution $u\left(\cdot, p_{k}\right)$ of (4.2)-(4.4), and a corresponding nontrivial solution of (4.5), (4.6).

This, in turn, will be done by showing that if $k \geqq k_{0}$, there are points $\tilde{p}_{k}$ and $\tilde{q}_{k}$ in the domain of $T_{N}$ for which both of the following inequalities hold:

$$
\sup \left\{\left\langle L_{k}^{\tilde{p}_{k}} \phi, \phi\right\rangle: \phi \in \Phi_{\tilde{p}_{k}},\|\phi\|^{2}=1\right\}<0,
$$

and

$$
\sup \left\{\left\langle L_{k}^{\tilde{q}_{k}} \phi, \phi\right\rangle: \phi \in \Phi_{\tilde{q}_{k}},\|\phi\|^{2}=1\right\}>0 .
$$

If we prove that the domain of $T_{N}$ is connected, then since the principal eigenvalue depends continuously on $p$ [as follows from (4.10)], we can conclude from (4.11) and (4.12) that there is a point $p_{k} \in \operatorname{dom}\left(T_{N}\right)$ for which

$$
\sup \left\{\left\langle L_{k}^{p_{k}} \phi, \phi\right\rangle: \phi \in \Phi_{p_{k}},\|\phi\|^{2}=1\right\}=0 .
$$

The corresponding eigenfunction, call it $a_{k}$, will be the desired non-trivial element in the kernel of the linearized operator $L_{k}^{p_{k}}$. To carry out this program will require a rather careful study of the properties of the time map $T_{N}$.

Let $f(u)$ be a smooth function defined on $\mathbb{R}$ which satisfies the following three conditions (cf. Fig. 2):

$$
f(u)=u \quad \text { if } u \leqq 1 ;
$$

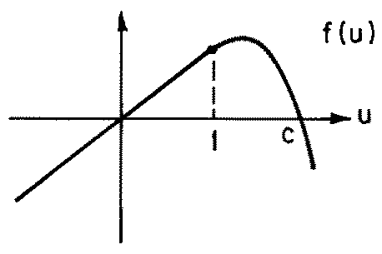


for some $c>1, f(u)>0$ on $[1, c), f(c)=0$, and $f^{\prime}(c)<0$;

$$
f^{\prime \prime}(u) \leqq 0 \text { for all } u \in[1, c] .
$$

Later on we shall further restrict $f$; the above conditions will suffice for our first few results.

Lemma 4.1. Let $f$ be defined as above. Then there is a positive integer $k_{0}$, and a point $p>0$, such that the solution $u(\cdot, p)$ of $(4.2),(4.3)$ exists, and for all integers $k \geqq k_{0}$,

$$
\sup \left\{\left\langle L_{k}^{p} \phi, \phi\right\rangle: \phi \in \Phi_{p},\|\phi\|^{2}=1\right\} \leqq 0 .
$$

Note that if equality holds in (4.16), there is nothing further to prove; otherwise we have that (4.11) holds with $\tilde{p}_{k}=p$ for all $k \geqq k_{0}$.

Proof. For balls of small radius $R$, the only solution of (4.2), (4.3) are the trivial solutions $u \equiv 0$, and $u \equiv c$. As $R$ increases, there is a critical value $\bar{R}$ for which a nonzero radial solution bifurcates out of 0 (see [8, Chap. 24]). Choose the positive integer $k_{0}$ to be the smallest one for which [see (2.11)], $\vec{R}^{2} \leqq-\lambda_{k_{0}}$; i.e.,

$$
k_{0}=\min \left\{k \in \mathbb{Z}_{+}: \bar{R}^{2} \leqq-\lambda_{k}\right\} \text {. }
$$

Note that if $k>k_{0}$, then $\bar{R}^{2}<-\lambda_{k}$.

Now for any $p, 0<p<1$, since $f$ is linear in the range $u \leqq p$, we see that we can (easily) find a solution of (4.2), (4.3) for which $T_{N}(p)=\bar{R}$. Hence if $\phi \in \Phi_{p}$ and $k \geqq k_{0}$, then

$$
\begin{aligned}
\left\langle L_{k}^{p} \phi, \phi\right\rangle & =\int_{0}^{\bar{R}}\left\{-\left(\phi^{\prime}\right)^{2}+\left(f^{\prime}(u(r, p))+\frac{\lambda_{k}}{r^{2}}\right) \phi^{2}\right\} r^{n-1} d r \\
& \leqq \int_{0}^{\bar{R}}\left(1+\frac{\lambda_{k}}{r^{2}}\right) \phi^{2} r^{n-1} d r \leqq 0,
\end{aligned}
$$

in view of (4.17).

We turn our efforts now to proving the far more difficult inequality (4.12); this will follow from a sequence of lemmas. [Recall that $T_{D}$ and $T_{N}$ are defined in (2.6).]

Lemma 4.2 (i) $\operatorname{dom}\left(T_{N}\right)=\operatorname{dom}\left(T_{D}\right)=(0, c)$.

(ii) If $1<p<c$, and $\tau(p)$ is defined by $u(\tau(p), p)=1$, then $\tau(p) \rightarrow \infty$ as $p \rightarrow c$.

Proof. If $p \in \operatorname{dom}\left(T_{D}\right)$, then since $f(0)=0$, we have from a result in [7], that $u^{\prime}\left(T_{D}(p), p\right)<0$. Thus for every such $p$, there is an $\varepsilon=\varepsilon(p)>0$ for which $u\left(T_{D}(p)\right.$ $+\varepsilon, p)<0$. Now on this solution, if $r>T_{D}(p)+\varepsilon$, then in the region $u^{\prime}<0, f(u(r))$ is bounded away from zero; say $f(u(r)) \leqq-\sigma^{2}$, if $r>T_{D}(p)+\varepsilon=T_{1}$. In this range, we may write

$$
\left(r^{n-1} u^{\prime}=-r^{n-1} f(u) \geqq r^{n-1} \sigma^{2},\right.
$$

and integrating from $T_{1}$ to $r$ gives

$$
u^{\prime}(r) \geqq\left(\frac{T_{1}}{r}\right)^{n-1}\left(u^{\prime}\left(T_{1}\right)-\frac{\sigma^{2} T_{1}}{n}\right)+\frac{\sigma^{2}}{n} r .
$$


This implies that $u^{\prime}(r)=0$ for some $r \geqq T_{1}$, and thus $p \in \operatorname{dom}\left(T_{N}\right)$. Hence $\operatorname{dom}\left(T_{D}\right)$ $C \operatorname{dom}\left(T_{N}\right)$, and since the reverse inclusion is obvious, we see that $\operatorname{dom}\left(T_{D}\right)$ $=\operatorname{dom}\left(T_{N}\right)$.

Next, we shall show that $\operatorname{dom}\left(T_{D}\right)=(0, c)$. Thus, if $0<p \leqq 1$, then since $f(u)=u$ on $0 \leqq u \leqq 1$, it is easy to see that $p \in \operatorname{dom}\left(T_{D}\right)$. Suppose now that $1<p<c$. On the interval $1 / 2<u<p, f(u)$ is bounded from below, and so as follows from [6, Lemma 6], the orbit of (4.28) starting at the point $u=p, v=0$, crosses the line $u=1 / 2$ in the region $v<0$. Define $\theta$ by $\tan \theta=v / u$; then if $r>\tau(p), f(u)=u$ and

$$
\theta^{\prime}=-1-\frac{n-1}{2 r} \sin 2 \theta
$$

Therefore for large $r$, say $r \geqq r_{0}, \theta^{\prime} \leqq-\frac{1}{2}$ so $\theta(r) \leqq \theta\left(r_{0}\right)-\frac{1}{2}\left(r-r_{0}\right)$, and thus $\theta(r)$ $<-\pi / 2$ for large $r$. It follows that there must be an $\bar{r}>0$ for which $\theta(\bar{r})=-\pi / 2$. Thus $u(\bar{r}, p)=0$ and $p \in \operatorname{dom}\left(T_{D}\right)$. This completes the proof of (i).

To see that $\tau(p) \rightarrow \infty$ as $p \rightarrow c$, we note that since $u(r, c)=c$ for all $r$, it follows by continuity, that given any $T>0$ and $\delta>0$, there is a neighborhood $U$ of $c$, such that if $p \in U$, then $|u(r, p)-c|<\delta$ for $r \leqq T$. Thus $\tau(p)>T$, and so $\tau(p) \rightarrow \infty$ as $p \rightarrow c$. This proves (ii) and completes the proof of the lemma.

For $p>1$, and $p$ near $c$, we define the three numbers $\tau(p), \sigma(p)$, and $s(p)$ by

$$
u(\tau(p), p)=1, \quad u(\sigma(p), p)=1 / 2,
$$

and

$$
s(p)=u\left(T_{N}(p), p\right) \text {. }
$$

Then obviously we have $\tau(p)<\sigma(p)<T_{D}(p)<T_{N}(p)$.

At this point we shall put some more conditions on $f$, in order to help us to make the technical details a little easier. To this end, let $F$ be the primative of $f$ defined by

$$
F^{\prime}=f, \quad F(0)=0 .
$$

We choose $c>1$ so close to 1 in order that

$$
\arcsin (1 / \sqrt{2 \mathrm{~F}(\mathrm{c})})>1+\pi / 6
$$

Next, let $\varepsilon$ be a positive number satisfying

$$
2\left(\frac{\pi}{6}-\varepsilon\right)>1+\varepsilon
$$

For such an $\varepsilon$, we want $c$ to also satisfy ${ }^{8}$

$$
\arcsin (1 / 2 \sqrt{2 F(c)})>\frac{\pi}{6}-\varepsilon
$$

Note that inequalities (4.20) and (4.22) are actually conditions which imply that $F(c)$ is close to $F(1)$.

\footnotetext{
${ }^{8}$ If we set $\phi(u)=\arcsin (2 F(u))^{-1 / 2}$, then $\phi(1)=\pi / 2$, since $F(1)=1 / 2$. But as $\pi / 2>1+\pi / 6$, we see that (4.20) can be achieved. If $\psi(u)=\arcsin (1 / 2 \sqrt{2 F(u)})$, then $\psi(1)=\pi / 6, \psi^{\prime}<0$, and thus (4.22) can be simultaneously achieved
} 
In what follows, we assume that $f$ is a fixed function which satisfies our earlier conditions (4.13)-(4.15), as well as (4.20)-(4.22). For such an $f$, we have the following proposition.

Proposition 4.3. Assume that $f$ satisfies (4.13)-(4.15), as well as (4.20)-(4.22), and let $k$ be a given positive integer, $k \geqq k_{0}$. Then there exists an interval $I=(c-\eta, c)$ such that both $\sigma(p)-\tau(p)$ and $T_{N}(p)-\sigma(p)$ are bounded from above on I. If there is $a \delta>0$ such that

$$
\left(T_{N}(p)-\sigma(p)\right)(\sigma(p)-\tau(p)) \geqq 1+\delta, \quad p \in I,
$$

then there is a function $\phi \in \Phi_{p}$ for which $\left\langle L_{k}^{p} \phi, \phi\right\rangle>0$.

Proof. In view of Lemma (4.2), if $p$ is close to $c, p<c$, then solutions of (4.2), (4.3) exist, and thus the quantities $\tau(p), \sigma(p), T_{D}(p)$, and $T_{N}(p)$ are all defined. We choose a function $\phi(r)$ as follows (see Fig. 3):

i) $\phi(r)=0$, if $r \leqq \tau(p)$,

ii) $\phi(r)=1$, if $r \geqq \sigma(p)$,

iii) $\phi$ is linear on $\tau(p) \leqq r \leqq \sigma(p)$.

Fig. 3

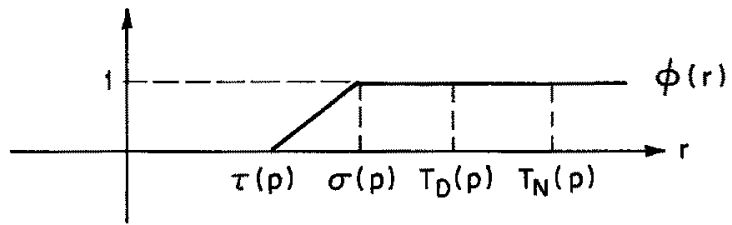

For this $\phi$, we have, (dropping the dependence on $p$ for notational convenience),

$$
\begin{aligned}
\int_{0}^{T_{N}}\left(f^{\prime}(u)+\frac{\lambda_{k}}{r^{2}}\right) \phi^{2} r^{n-1} d r & =\int_{\tau}^{\sigma}\left(f^{\prime}(u)+\frac{\lambda_{k}}{r^{2}}\right) \phi^{2} r^{n-1} d r+\int_{0}^{T_{N}}\left(f^{\prime}(u)+\frac{\lambda_{k}}{r^{2}}\right) \phi^{2} r^{n-1} d r \\
& \geqq \int_{0}^{T_{N}}\left(f^{\prime}(u)+\frac{\lambda_{k}}{r^{2}}\right) r^{n-1} d r,
\end{aligned}
$$

since if $\tau \leqq r \leqq \sigma$, we have by the last lemma,

$$
f^{\prime}(u(r))+\frac{\lambda_{k}}{r^{2}}=1+\frac{\lambda_{k}}{r^{2}} \geqq 1+\frac{\lambda_{k}}{\tau^{2}} \geqq 0,
$$

for $p$ near $c$. Thus,

$$
\begin{aligned}
& \int_{0}^{T_{N}}\left(f^{\prime}(u)+\frac{\lambda_{k}}{r^{2}}\right) \phi^{2} r^{n-1} d r \\
& \quad \geqq \int_{\sigma}^{T_{N}}\left(1+\frac{\lambda_{k}}{r^{2}}\right) r^{n-1} d r=\frac{T_{N}^{n}-\sigma^{n}}{n}+\lambda_{k}\left(\frac{T_{N}^{n-2}-\sigma^{n-2}}{n-2}\right) \\
& \quad=\left(T_{N}-\sigma\right)\left\{\frac{T_{N}^{n-1}+T_{N}^{n-2} \sigma+\ldots+\sigma^{n-1}}{n}+\lambda_{k} \frac{T_{N}^{n-3}+T_{N}^{n-4} \sigma+\ldots+\sigma^{n-3}}{n-2}\right\},
\end{aligned}
$$

so that for $p$ near $c$,

$$
\int_{0}^{T_{N}}\left(f^{\prime}(u)+\frac{\lambda_{k}}{r^{2}}\right) \phi^{2} r^{n-1} d r \geqq\left(T_{N}-\sigma\right)\left(\sigma^{n-1}+\lambda_{k} T_{N}^{n-3}\right) .
$$


Next, since we may approximate $\phi$ in $L_{2}\left(0, T_{N}(p)\right)$ as close as we please by a smooth function, we may assume that $\phi \in C^{1}$ in evaluating $\left\langle L_{k}^{p} \phi, \phi\right\rangle$. This gives

$$
\begin{aligned}
\int_{0}^{T_{N}}-\left(\phi^{\prime}\right)^{2} r^{n-1} d r & =\int_{\tau}^{\sigma}-\frac{1}{(\sigma-\tau)^{2}} r^{n-1} d r=-\frac{1}{(\sigma-\tau)^{2}}\left(\frac{\sigma^{n}-\tau^{n}}{n}\right) \\
& =-\frac{1}{\sigma-\tau}\left(\frac{\sigma^{n-1}+\sigma^{n-2} \tau+\ldots+\tau^{n-1}}{n}\right) .
\end{aligned}
$$

Therefore,

$$
\int_{0}^{T_{N}}-\left(\phi^{\prime}\right)^{2} r^{n-1} d r \geqq-\frac{\sigma^{n-1}}{\sigma-\tau} .
$$

If we add (4.24) and (4.25), we get

$$
\begin{aligned}
\left\langle L_{k}^{p} \phi, \phi\right\rangle & \geqq \sigma^{n-1}\left[\left(T_{N}-\sigma\right)-\frac{1}{(\sigma-\tau)}\right]+\lambda_{\mathrm{K}} T_{N}^{n-3}\left(T_{N}-\sigma\right) \\
& =\sigma^{n-1}\left[\frac{\left(T_{N}-\sigma\right)(\sigma-\tau)-1}{\sigma-\tau}\right]+\lambda_{k} T_{N}^{n-3}\left(T_{N}-\sigma\right) .
\end{aligned}
$$

Now we shall show that there are constants $A>0$ and $B>0$ such that for $p$ near $c, p<c$,

$$
\sigma(p)-\tau(p) \leqq A \quad \text { and } \quad T_{N}(p)-\sigma(p) \leqq B .
$$

Granting these, we find from (4.26), and (4.23), that

$$
\left\langle L_{k}^{p} \phi, \phi\right\rangle \geqq \frac{\sigma^{n-1} \delta}{A}+\lambda_{k} T_{N}^{n-3} B \geqq \frac{\sigma^{n-1} \delta}{A}+\lambda_{k} B(\sigma+B)^{n-3} .
$$

But $\sigma(p)>\tau(p)$, and since $\tau(p) \rightarrow \infty$ as $p \rightarrow c$ (by Lemma 4.2), we see that for $p$ near $c$, $(\sigma+B)^{n-3} \leqq 2^{n-3} \sigma^{n-3}$. Therefore, for $p$ near $c$

$$
\left\langle L_{k}^{p} \phi, \phi\right\rangle \geqq \sigma^{n-3}\left(\sigma^{2} \frac{\delta}{A}+\lambda_{k} B 2^{n-3}\right)>0,
$$

as desired. Thus in order to complete the proof, it suffices to show that both $\sigma(p)$ $-\tau(p)$, and $T_{N}(p)-\sigma(p)$ are bounded from above, for $p$ near $c$.

To this end, suppose that $\frac{1}{2}<u \leqq 1$; then on this range

$$
-\left(r^{n-1} u^{\prime}\right)^{\prime}=r^{n-1} f(u)=r^{n-1} u \geqq \frac{1}{2} r^{n-1} .
$$

Integrating from $\tau$ to $r \leqq \sigma$ gives,

so that for $\tau \leqq r \leqq \sigma$,

$$
-r^{n-1} u^{\prime}(r) \geqq-r^{n-1} u^{\prime}(r)+\tau^{n-1} u^{\prime}(\tau) \geqq \frac{1}{2 n}\left(r^{n}-\tau^{n}\right),
$$

$$
-u^{\prime}(r) \geqq \frac{r}{2 n}-\frac{\tau^{n} r^{1-n}}{2 n}>\frac{r-\tau}{2 n} .
$$

If we integrate this from $\tau$ to $\sigma$, we get

$$
\frac{1}{2}=-u(\sigma)+u(\tau) \geqq \frac{(\sigma-\tau)^{2}}{4 n},
$$


and thus

$$
\sqrt{2 n} \geqq \sigma-\tau
$$

Next, for $p$ near $c$, if we write (2.3) as the first-order system

$$
u^{\prime}=v, \quad v^{\prime}=-\frac{n-1}{r} v-f(u),
$$

and define

$$
H(r, p) \equiv \frac{v^{2}(r, p)}{2}+F(u(r, p))
$$

we find that $H_{r}=(1-n) v^{2} / r \leqq 0$. Thus along the orbit of $(4.28)$ which starts at the point $u=p, v=0$ when $r=0$, we have (cf. (4.19)),

$$
F(s)=H\left(T_{N}, p\right) \leqq H(r, p)=F(u)+v^{2} / 2 .
$$

So if $r \geqq \sigma$ (i.e., $u(r, p) \leqq 1$ ), we obtain

$$
\sqrt{s^{2}-u^{2}}=\sqrt{2(F(s)-F(u))} \leqq-v .
$$

Now we shall show in the lemma below that for $p$ close to $c, s(\mathrm{p})<-1 / 2$. Granting this, we obtain

$$
T_{N}-\sigma=\int_{1 / 2}^{s} \frac{d u}{v}=\int_{s}^{1 / 2} \frac{d u}{-v} \leqq \int_{s}^{1 / 2} \frac{d u}{\sqrt{s^{2}-u^{2}}}=\arcsin \frac{1}{2|s|}-\pi / 2 \leqq \pi,
$$

and this completes the proof of the propostion.

Lemma 4.4. For $p$ close to $c, p<c$, the function $s(p)$, defined by (4.19) satisfies

$$
s(p)<-1 / 2 \text {. }
$$

Proof. Choose $\eta>0$ so small that

$$
F(c)-2 \eta>1 / 8
$$

This can be done since $F(c)>F(1)=\frac{1}{2}$. Now orbits of $(4.28)$ which start at the point $u=p, v=0$ at $r=0,(0<p<c)$, all lie inside the closed level curve of $H$ defined by $\frac{v^{2}}{2}$ $+F(u)=F(c)$. Thus, there are constants $M>0$, and $d>0$ such that along each such orbit, we have the bounds

$$
-d^{2} \leqq u(r, p)<c, \quad \text { and } \quad 0 \leqq-u^{\prime}(r, p) \leqq M, \quad \text { if } \quad 0 \leqq r \leqq T_{N}(p),
$$

for all $p \in I \equiv(0, c)$.

Now let $T>(n-1) M\left(c+d^{2}\right) / \eta$; then for $p$ near $c$, and $s(p)<u(0, p)<c$, we claim that ${ }^{9}$

$$
H(T, p)-H(r, p)<\eta, \text { if } \quad T \leqq r \leqq T_{N}(p) .
$$

\footnotetext{
${ }^{9}$ Since $T_{N}(p) \rightarrow \infty$ as $p \nearrow c$, such a $T<T_{N}(p)$ exists if $p$ is near $c$
} 
Indeed,

$$
\begin{aligned}
H(T, p)-H(r, p) & =\int_{T}^{r}-H^{\prime}(t, p) d t=\int_{T}^{r}(n-1) \frac{u^{\prime}(t, p)^{2}}{t} d t \\
& \leqq \frac{(n-1) M}{T} \int_{T}^{r}-u^{\prime}(t, p) d t=\frac{(n-1) M}{T}(u(T, p)-u(r, p)) \\
& \leqq \frac{(n-1) M}{T}\left(c+d^{2}\right)<\eta .
\end{aligned}
$$

Since $u^{\prime}(r, c)=0$ for all $r$, we have $H(r, c)=F(c)$ for all $r$. Since $H$ is continuous, there is a $\xi>0$ such that $H(T, c)-H(T, p)<\eta$ if $c-\xi<p<c$. Thus, if $c-\xi<p<c$, we have, for $T \leqq r \leqq T_{N}(p)$,

$$
H(r, p) \geqq H(T, p)-\eta>H(T, c)-2 \eta=F(c)-2 \eta>\frac{1}{8} .
$$

It follows that $s^{2} / 2=H\left(T_{N}(p), p\right)>\frac{1}{8}$, and this gives (4.30).

We can now state the following theorem, which establishes infinitesimal symmetry-breaking where the corresponding null-spaces of the associated linearized operators have arbitrarily high dimensions.

Theorem 4.5. Let $f$ satisfy (4.13)-(4.15), as well as (4.20)-(4.22), and let $k_{0}$ be defined as in (4.17). Then there exists a sequence of points $\left\{p_{k}: k \geqq k_{0}\right\} \subset(0, c)$ such that the symmetry breaks infinitesimally on each radial solution $u\left(\cdot, p_{k}\right)$ of $(4.2)-(4.4)$, $\left(p=p_{k}\right)$. Furthermore, the kernel of the linearized operator $L_{k}^{p_{k}}(c f .(2.8))$, has dimension $\left(\begin{array}{c}k+n-2 \\ k\end{array}\right) \cdot\left(\frac{2 k+n-2}{k+n-2}\right)$.

Proof. In view of Lemma 4.1, and Proposition 4.3, we must show two things; namely, that (4.23) holds for all $p$ near $c, p<c$, and that the domain of $T_{N}$ is connected. However, the connectedness of $\operatorname{dom}\left(T_{N}\right)$ follows from Lemma 4.2. Thus we need only show (4.23). For $0<p<c$, we have $H(r, p) \leqq H(0, p)=F(p)<F(c)$, so that if $1 / 2 \leqq u(r, p) \leqq 1$, and $v=u^{\prime}$,

$$
-v \leq \sqrt{2(F(c)-F(u))}=\sqrt{\tilde{c}^{2}-u^{2}}
$$

where $\tilde{c}^{2}=2 F(c)$. Thus from $(4.20)$,

$$
\begin{aligned}
\sigma(p)-\tau(p) & =\int_{1}^{1 / 2} \frac{d u}{v}=\int_{1 / 2}^{1} \frac{d u}{-v} \geqq \int_{1 / 2}^{1} \frac{d u}{\sqrt{\tilde{c}^{2}-u^{2}}} \\
& =\arcsin \frac{1}{\tilde{c}}-\arcsin \frac{1}{2 \tilde{c}}=\arcsin \frac{1}{\sqrt{2 F(c)}}-\arcsin \frac{1}{2 \sqrt{2 F(c)}} \\
& >\left(1+\frac{\pi}{6}\right)-\frac{\pi}{6}
\end{aligned}
$$
because $\tilde{c}>1$ implies that $\arcsin \frac{1}{2 \tilde{c}} \leqq \arcsin \frac{1}{2}=\pi / 6$. Therefore for $1<p<c$, we
have

$$
\sigma(p)-\tau(p)>1
$$


Next, if $u(r, p) \leqq 1$, then (4.34) holds so using (4.30) we have, for $1<p<c$, $p$ near $c$,

$$
\begin{aligned}
T_{N}(p)-\sigma(p) & =\int_{s}^{1 / 2} \frac{d u}{-v} \geqq \int_{s}^{1 / 2} \frac{d u}{\sqrt{\tilde{c}^{2}-u^{2}}} \\
& =\arcsin \left(\frac{1}{2 \tilde{c}}\right)-\arcsin \left(\frac{s}{\tilde{c}}\right) \geqq \arcsin \left(\frac{1}{2 \tilde{c}}\right)+\arcsin \left(\frac{1}{2 \tilde{c}}\right) \\
& =2 \arcsin \left(\frac{1}{2 \tilde{c}}\right)>2\left(\frac{\pi}{6}-\varepsilon\right)>1+\varepsilon,
\end{aligned}
$$

in view of (4.21) and (4.22). This, together with (4.35) implies (4.23). The proof of the theorem is complete.

We shall next obtain some further information on the sequence of points $\left\{p_{k}\right\}$ whose existence was proved in the last theorem.

Theorem 4.6. One can choose a sequence of points $\left\{p_{k}: k \geqq k_{0}\right\} \subset(0, c)$ converging monotonically to $c$, and such that the $p_{k}$ satisfy the conclusion of the last theorem.

Proof. If $k \geqq k_{0}$, then by construction we have

$$
\sup \left\{\left\langle L_{k}^{p_{k}} \phi, \phi\right\rangle: \phi \in \Phi_{p_{k}},\|\phi\|^{2}=1\right\}=0 .
$$

Using (2.10) we can write $\lambda_{k+1}=\lambda_{k}-(2 k+n-1)$, so that

$$
f^{\prime}\left(u\left(r, p_{k}\right)\right)+\frac{\lambda_{k+1}}{r^{2}}=f^{\prime}\left(u\left(r, p_{k}\right)\right)+\frac{\lambda_{k}}{r^{2}}-\frac{(2 k+n-1)}{r^{2}},
$$

if $0<r \leqq T_{N}\left(p_{k}\right)$. Thus if $\phi \in \Phi_{p_{k}}$,

$$
\begin{aligned}
\left\langle L_{k+1}^{p_{k}} \phi, \phi\right\rangle & =\int_{0}^{T_{N}\left(p_{k}\right)}\left\{-(\phi)^{2}+\left[f^{\prime}\left(u\left(r, p_{k}\right)\right)+\frac{\lambda_{k}+1}{r^{2}}\right] \phi^{2}\right\} r^{n-1} d r \\
& =\left\langle L_{k}^{p_{k}} \phi, \phi\right\rangle-\int_{0}^{T_{N}\left(p_{k}\right)} \frac{2 k+n-1}{r^{2}} r^{n-1} \phi^{2} d r<0,
\end{aligned}
$$

in view of (4.36).

It follows that

$$
\sup \left\{\left\langle L_{k+1}^{p_{k}} \phi, \phi\right\rangle: \phi \in \Phi_{p_{k}},\|\phi\|^{2}=1\right\} \leqq 0
$$

If this supremum were equal to zero, it would mean that we could find a non-zero function $a_{k+1}$ which would satisfy the equation

$$
a^{\prime \prime}(r)+\frac{n-1}{r} a^{\prime}(r)+\left(f^{\prime}\left(u\left(r, p_{k}\right)\right)+\frac{\lambda_{k+1}}{r^{2}}\right) a(r)=0, \quad 0<r<T_{N}\left(p_{k}\right)
$$

together with the boundary conditions

$$
a(0)=a^{\prime}\left(T_{N}\left(p_{k}\right)\right)=0 .
$$

However since $a_{k} \neq 0$, this would violate Corollary 3.6. It follows that strict inequality holds in (4.37). Thus $p_{k+1}$ may be chosen so as to achieve $p_{k+1}>p_{k}$. 
Next, recall that $p_{k}<c$ for all $k$, so that if $p_{k} \rightarrow \bar{p}<c$, as $k \rightarrow \infty$, we would have $T_{N}\left(p_{k}\right) \rightarrow T_{N}(\tilde{p})<\infty$, and thus the sequence $\left\{T_{N}\left(p_{k}\right): k \geqq k_{0}\right\}$ would be bounded; say $T_{N}\left(p_{k}\right) \leqq M$, for all $k \geqq k_{0}$. Now using (4.36), we know that for each $k \geqq k_{0}$, there exists $\phi_{k} \in \Phi_{p_{k}}$, satisfying $\left\langle L_{k}^{p_{k}} \phi_{k}, \phi_{k}\right\rangle=0$, and $\left\|\phi_{k}\right\|^{2}=1$. For such $\phi_{k}$, we have

$$
\begin{aligned}
\int_{0}^{T_{N}\left(p_{k}\right)} \frac{1}{r^{2}} \phi_{k}(r)^{2} r^{n-1} d r & \geqq \frac{1}{M^{2}} \int_{0}^{T_{N}\left(p_{k}\right)} \phi_{k}(r)^{2} r^{n-1} d r \\
& =\frac{1}{M^{2}}\left\|\phi_{k}\right\|^{2}=\frac{1}{M^{2}} .
\end{aligned}
$$

On the other hand $\left\langle L_{k}^{p_{k}} \phi_{k}, \phi_{k}\right\rangle=0$ and $f^{\prime} \leqq 1$ imply that

$$
\begin{aligned}
0 & \leqq \int_{0}^{T_{N}\left(p_{k}\right)} \phi_{k}^{\prime}(r)^{2} r^{n-1} d r \\
& =\int_{0}^{T_{N}\left(p_{k}\right)}\left[f^{\prime}\left(u\left(r, p_{k}\right)\right)\right] \phi_{k}(r)^{2} r^{n-1} d r+\int_{0}^{T_{N}\left(p_{k}\right)} \frac{\lambda_{k}}{r^{2}} \phi_{k}(r)^{2} r^{n-1} d r \\
& \leqq \int_{0}^{T_{N}\left(p_{k}\right)} \phi_{k}(r)^{2} r^{n-1} d r+\lambda_{k} \int_{0}^{T_{N}\left(p_{k}\right)} \frac{1}{r^{2}} \phi_{k}(r)^{2} r^{n-1} d r .
\end{aligned}
$$

It follows from (4.38) that

$$
0 \leqq 1+\frac{\lambda_{k}}{M^{2}}
$$

for all $k \geqq k_{0}$. This is impossible, and the proof is complete. $\square$

Observe now that if $\bar{f}$ is $C^{1}$-close to $f$, then it is easy to see that the corresponding time maps $\bar{T}_{N}, \bar{T}_{D}, \bar{\sigma}, \bar{\tau}$ for $\bar{f}$ are $L^{\infty}$ close to the corresponding time maps $T_{N}, T_{D}, \sigma, \tau$, of $f$, at least on compact intervals. Also since the inequalities (4.23) and (4.30) are "open" conditions, and since Lemma 4.2 continues to hold for $\bar{f}$, we see that Theorems 4.5 and 4.6 continue to hold for such $\bar{f}$. Since we shall make use of this fact later, we state it formally.

Theorem 4.7. There is an open neighborhood of $f$ in the $C^{1}$-topology for which Theorems 4.5 and 4.6 are valid.

\section{Symmetry-Breaking in the Neumann Problem}

We turn our attention now to the task of showing that the symmetry actually breaks at each of the points $p_{k}$; more precisely, (in order that our "bifurcation diagram" has a nice form), we shall actually construct a function $\bar{f}$ which is $C^{1}$ close to the given $f$, for which the symmetry-breaks at each of the corresponding points $\bar{p}_{k}$. In order to do this, we shall rely on the bifurcation theorem stated in Sect. 2; Theorem 2.2 .

Thus we may assume that (4.2), (4.3) has solutions for all $p, 0<p<c$. We know that for the given $f$, there is a real number $\varrho_{1}>0$ such that the set

$$
\left\{u(r, p): 0 \leqq r \leqq T_{N}(p), 0<p<c\right\},
$$


is contained in the interior of the interval $|u|<\varrho_{1}$. In what follows, we shall drop the "bars," and assume that $f$ is a function which satisfies the hypotheses and conclusion of Theorem 4.8, as well as the condition

$$
(f(u) / u)^{\prime} \leqq 0, \quad|u|<\varrho,
$$

where $\varrho>2 \varrho_{1}$.

Proposition 5.1. If $0<p<c$, then

$$
T_{N}^{\prime}(p)>0 .
$$

In particular, no radial bifurcation is possible.

Proof. Inequality (5.2) is a consequence of (5.1), as follows from arguments similar to those given in [6, Theorems 2 and 3]. The last sentence follows from the remark given after the statement of Theorem 3.8. becomes

In order to apply Theorem 2.2, we first make a change of scale so that (2.1)

$$
\lambda \Delta u(x)+f(u(x))=0, \quad x \in D_{1}^{n},
$$

together with the boundary condition, $d u / d n=0$ on $|x|=1$. Here $\lambda\left(=R^{-2}\right)$ is now to be thought of as the bifurcation parameter. We let $B$ be the space of real valued $H^{1}$ functions $u$ on $D_{1}^{n}$ which satisfy homogeneous Neumann boundary conditions, and which are $O(n-1)$-invariant; i.e. if $T \in O(n-1)$, then $u\left(T \bar{x}, x_{n}\right)=u\left(\bar{x}, x_{n}\right)$, where $\bar{x}=\left(x_{1}, \ldots, x_{n-1}\right)$.

We endow $B$ with the following inner-product:

$$
\langle u, v\rangle=\int_{D_{1}^{n}} \nabla u \nabla v+\int_{S^{n-1}} u v .
$$

Now take any (fixed) $p_{k}$, and let $\lambda_{0}=T_{N}\left(p_{k}\right)^{-2}$. We implicitly define an operator $A: B \rightarrow B$ by

$$
\langle A(u), v\rangle=\int_{D_{1}^{n}}\left(f\left(u+u_{0}\right)-f\left(u_{0}\right)\right) v+\lambda_{0} \int_{S^{n-1}} u v, \quad \forall v \in B,
$$

where, in this new parametrisation, $u_{0}$ is a radial solution of the Neumann problem: $\lambda_{0} \Delta u_{0}+f\left(u_{0}\right)=0$; i.e., $u_{0}(\cdot)=u\left(\cdot, p_{k}\right)$. Next we define $F: B \times \mathbb{R} \rightarrow B$ by $F(u, \lambda)=A(u)-\lambda u$, and set $L_{0}=F_{u}\left(0, \lambda_{0}\right) \equiv A^{\prime}(0)-\lambda_{0} I, L_{1}=F_{\lambda u}\left(0, \lambda_{0}\right) \equiv-I$.

For any space $W$ of functions we denote by $W^{o(n-1)}$ to be those functions in $W$ which are invariant under $O(n-1)$. Let $\tilde{\Delta}$ denote the Laplacian (LaplaceBeltrami operator) on $S^{n-1}$, and set

$$
V=\left\{w \in H^{1}\left(S^{n-1}\right): \tilde{\Delta} w=\lambda_{0} w\right\}^{o(n-1)} .
$$

We now have the following proposition whose proof we defer to the appendix.

Proposition 5.2. $V$ is a one-dimensional space.

We can now verify the hypotheses of Theorem 2.2. (a): $L_{0}$ has a onedimensional kernel. 
Proof. We first calculate $A^{\prime}(0)$.

$$
\begin{aligned}
\left\langle A^{\prime}(0) z, v\right\rangle & =\lim _{t \rightarrow 0}\left\langle\frac{A(t z)-A(0)}{t}, v\right\rangle \\
& =\lim _{t \rightarrow 0} \frac{1}{t}\left\{\int_{D_{1}^{n}}\left[f\left(t z+u_{0}\right)-f\left(u_{0}\right)\right] v+t \lambda_{0} \int_{S^{n-1}} z v\right\} \\
& =\int_{D_{1}^{n}} f^{\prime}\left(u_{0}\right) z v+\lambda_{0} \int_{S^{n-1}} z v .
\end{aligned}
$$

Thus if $L_{0}(z)=0$, we have

$$
0=\left\langle A^{\prime}(0) z-\lambda_{0} z_{,} v\right\rangle=\int\left(f^{\prime}\left(u_{0}\right) z+\lambda_{0} \Delta z\right) v, \quad \forall v \in B
$$

thus we see that $z$ satisfies

$$
\lambda_{0} \Delta z+f^{\prime}\left(u_{0}\right) z=0
$$

so $z$ is of the form $a_{N}(n) \tilde{\Phi}_{N}(\theta)$, and Proposition 5.2 implies that $z$ is uniquely determined; this proves (a). Next, since $L_{0}$ is a Fredholm operator, we have that (a) implies (b). Finally we check (c): $L_{1} w$ is not in the range of $L_{0}$; here $L_{0}$ is spanned by $w$.

Proof. Suppose $L_{1} w=L_{0} z$; then $-w=A^{\prime}(0) z-\lambda_{0} z$. Thus for all $v \in B,\langle-w, v\rangle$ $=\left\langle A^{\prime}(0) z-\lambda_{0} z, v\right\rangle$, and after integrating by parts,

$$
\int_{D_{1}^{n}} v \Delta w-\int_{S^{n-1}} v w=\int_{D_{1}^{n}} f^{\prime}\left(u_{0}\right) z v-\lambda_{0} \int_{D_{1}^{n}}(\nabla z)(\nabla v)
$$

or

$$
\int\left(\Delta w-f^{\prime}\left(u_{0}\right) z-\lambda_{0} \Delta z\right) v=\int_{S^{n-1}} v w
$$

If we set $v=w$ in this last equation, we get

$$
\int_{D_{i}^{n}} w \Delta w-f^{\prime}\left(u_{0}\right) z w-\lambda_{0}(\Delta z) w=\int_{S^{n-1}} w^{2},
$$

and since $w$ satisfies the linearized equation, we find

$$
\int_{D_{1}^{n}}-(\nabla w)^{2}=\int_{S^{n-1}} w^{2}
$$

This yields the contradiction $w \equiv 0$. Hence all of the hypotheses of Theorem 2.2 hold. Thus, from this theorem, we see that if $B=[w] \oplus[w]^{\perp}$, there is a $\delta>0$ and functions $\lambda(s), u(s)=s(w+\phi(s))$, where $\phi \in[w]^{\perp}, \phi(0)=0, \lambda(0)=\lambda_{0}$ and $F(u(s)$, $\lambda(s)) \equiv 0,|s|<\delta$. It follows that $A(u(s))=\lambda(s) u(s)$, so that for all $v \in B,\langle A(u(s))$ $-\lambda(s) u(s), v\rangle=0$. Thus

$$
\int_{D_{1}^{n}}\left(f\left(u(s)+u_{0}\right)+\lambda_{0} \Delta u_{0}+\lambda(s) \Delta u(s)\right) v+\left(\lambda_{0}-\lambda(s)\right) \int_{s^{n-1}} u(s) v=0 .
$$

Thus, if the support of $v$ lies in $\left(D_{1}^{n}\right)^{\text {int }}$, this last equation yields

$$
\int_{D_{1}^{n}}\left[f\left(u+u_{0}\right)+\lambda_{0} \Delta u_{0}+\lambda \Delta u\right] v=0 \text {. }
$$


From this it follows that $u$ is a weak solution to the equation $f\left(u+u_{0}\right)+\lambda_{0} \Delta u_{0}$ $+\lambda \Delta u=0$, so that by the usual regularity theorems, $[0], u$ is a classical solution of this equation, up to the boundary. It follows that the term in brackets in (5.5) vanishes in $\overline{D_{1}^{n}}$, so from (5.4) we have for all $v \in B$,

$$
\left(\lambda_{0}-\lambda(s)\right) \int_{S_{n-1}} u(s) v=0 .
$$

If we put $v=w$, we find

$$
\left(\lambda_{0}-\lambda(s)\right) \int_{s^{n-1}}\left(w^{2}+w \phi(s)\right)=0 .
$$

Now since

$$
\int_{s^{n-1}} w^{2}=a_{N}(R)^{2} \int_{S^{n-1}} \Phi_{N}^{2} \neq 0,
$$

and $\phi(0)=0$, we see that for $s$ near 0 ,

$$
\int_{s^{n-1}}\left(w^{2}+w \phi(s)\right) \neq 0,
$$

and thus from (5.6), we find $\lambda(s) \equiv \lambda_{0}$ for $s$ near 0 . Since $f\left(u+u_{0}\right)+\lambda_{0} \Delta u_{0}+\lambda \Delta u=0$, we obtain

$$
f\left(u(s)+u_{0}\right)+\lambda_{0} A\left(u(s)+u_{0}\right)=0,
$$

for sufficiently small $s$. That is, $u_{0}+u(s)$ is a solution of our original equation, so that bifurcation actually occurs.

It follows that there is bifurcation at each radial solution $u\left(\cdot, p_{k}\right)$; in fact, since $T_{n}$ is monotone, Proposition 5.1 shows that the bifurcating solutions are asymmetric ones. But as Eq. (2.1) is $O(n)$-invariant, there bifurcates out of each radial solution $u\left(\cdot, p_{k}\right)$, at least an $n$-dimensional set of asymmetric solutions. This yields the following theorem.

Theorem 5.7. Let $f$ satisfy hypotheses (4.13)-(4.15), (4.20)-(4.22), and (5.1). Let $k_{0}$ be the positive integer defined by (4.17). Then

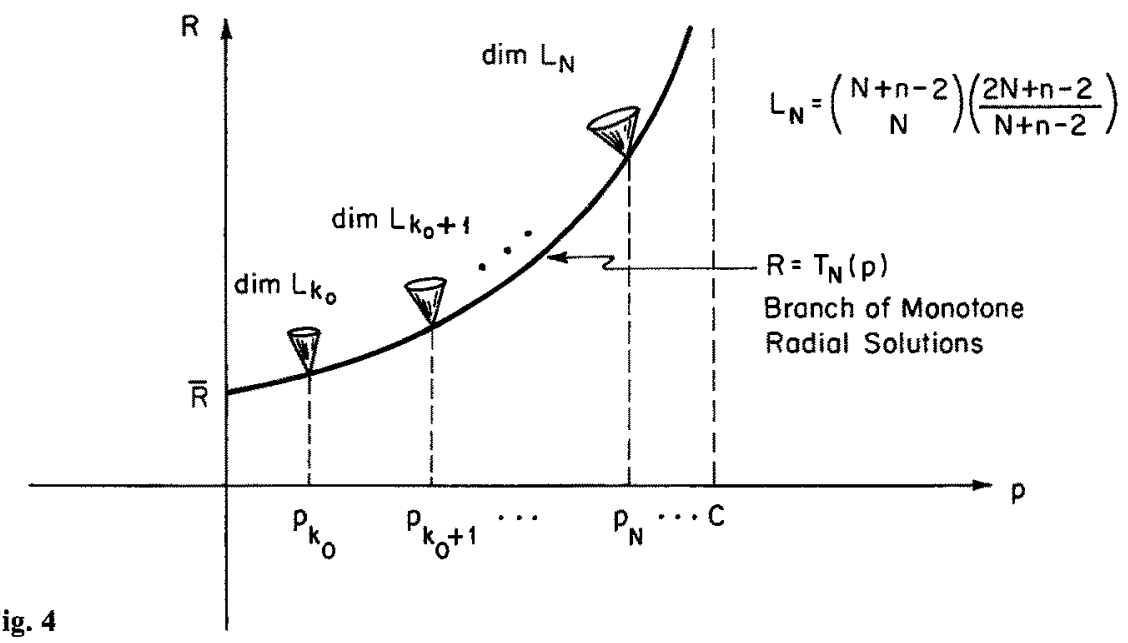



and

i) There exists an increasing sequence of points $\left\{p_{k}: k \geqq k_{0}\right\} \subset(0, c)$, with $p_{k} \rightarrow c$,

ii) There exists a corresponding sequence of monotone radial solutions $\left\{u\left(\cdot, p_{k}\right): k \geqq k_{0}\right\}$ of $(4.2),(4.3)$, such that on each solution $u\left(\cdot, p_{k}\right)$ the following hold:

a) the symmetry breaks,

b) the dimension of the set of bifurcating asymmetric solutions is at least $n$.

Notice that the kernel of the linearized operator at $u_{k}$ has dimension $\left(\begin{array}{c}k+n-2 \\ k\end{array}\right) \frac{2 k+n-2}{k+n-2}=L_{k}$, which is considerably larger than $n$. We conjecture that there bifurcates out of each $u_{k}$ an $L_{k}$-dimensional manifold of asymmetric solutions. If this were true, then the bifurcation diagram would be as depicted in the figure above.

\section{Appendix}

We give here the proof of Proposition 5.2.

Let $H_{k}$ denote the homogeneous, harmonic polynomials of degree $k$ in $x_{1}, \ldots, x_{n}$. It is well-known, (see [4]), that there is an isomorphism $r: H_{k} \rightarrow V_{k}$ $=\left\{\phi \in C^{2}\left(S^{n-1}\right): \tilde{\Delta} \phi=\lambda_{k} \phi\right\}$, given by $r(p)=\left.p\right|_{S^{n-1}}$, and it is easy to see that this map is $O(n)$-equivariant; i.e., $r(T p)=T(r p)$, or $\left.(p \circ T)\right|_{S^{n-1}}=\left(\left.p\right|_{S^{n-1}}\right) \circ T$. It follows that

$$
H_{k}^{o(n-1)} \cong V_{k}^{o(n-1)}
$$

Thus to prove the proposition, it suffices to show that $H_{k}^{O(n-1)}$ is one-dimensional.

Let $P_{n}$ denote the space of polynomials in $x=\left(x_{1}, \ldots, x_{n}\right)$.

Lemma 5.3. $P_{n}^{O(n)}=R\left[r^{2}\right] \equiv$ polynomials in $r^{2}=x_{1}^{2}+\ldots+x_{n}^{2}$.

Proof. By induction on $n$. If $n=1$, and $p \in P_{1}^{o(1)}$, then $p(x)=p(-x)$, so $p(x)=q\left(x^{2}\right)$ $=q\left(r^{2}\right)$. Now suppose that $P_{n-1}^{O(n-1)}=R\left[\tilde{r}^{2}\right]$, where $\tilde{r}=x_{1}^{2}+\ldots+x_{n-1}^{2}$. Let $p \in P_{n}^{O(n)}$; then $p(x)=a_{0}(\tilde{x})+a_{1}(\tilde{x}) x_{n}+\ldots+a_{k}(\tilde{x}) x_{n}^{k}$, where $\tilde{x}=\left(x_{1}, \ldots, x_{n-1}\right)$. But $p(\tilde{x}, 0)$ $=a_{0}(\tilde{x})$, and since $p \in P_{n}^{O(n)}, p \in P_{n}^{O(n-1)}$, so $a_{0}$ is $O(n-1)$-invariant. Now given $x=\left(x_{1}, \ldots, x_{n}\right)$, choose $T \in O(n)$ such that $T x=\left(y_{1}, y_{2}, \ldots, y_{n-1}, 0\right)$. Note that $x_{1}^{2}$ $+\ldots+x_{n}^{2}=y_{1}^{2}+\ldots+y_{n-1}^{2}$. Now

$$
p\left(x_{1}, \ldots, x_{n}\right)=p\left(T\left(x_{1}, \ldots, x_{n}\right)\right)=p\left(y_{1}, \ldots, y_{n-1}, 0\right)=a_{0}\left(y_{1}, \ldots, y_{n-1}\right) .
$$

But by our induction hypothesis, $a_{0}\left(y_{1}, \ldots, y_{n-1}\right)=q\left(y_{1}^{2}+\ldots+y_{n-1}^{2}\right)=q\left(x_{1}^{2}\right.$ $\left.+\ldots+x_{n}^{2}\right)$, where $q$ is a polynomial. This proves the lemma.

Lemma 5.4. $P_{n}^{O(n-1)}=R\left[r^{2}, x_{n}\right] \equiv$ polynomial ring spanned by $r^{2}=x_{1}^{2}+\ldots+x_{n}^{2}$, and $x_{n}$.

Proof. Let $p \in P_{n}^{O(n-1)}$; then if $x=\left(x_{1}, \ldots, x_{n}\right)$,

$$
p(x)=a_{0}\left(x_{1}, \ldots, x_{n-1}\right)+a_{1}\left(x_{1}, \ldots, x_{n-1}\right) x_{n}+\ldots+a_{k}\left(x_{1}, \ldots, x_{n-1}\right) x_{n}^{k} .
$$

We claim that each $a_{i}$ is $O(n-1)$ invariant. To see this, note that $p\left(x_{1}, \ldots, x_{n-1}, 0\right)$ $=a_{0}\left(x_{1}, \ldots, x_{n-1}\right)$ so as in Lemma 5.3, $a_{0}$ is $O(n-1)$ invariant. Thus $p-a_{0}$ is 
$O(n-1)$ invariant, and since

$$
p-a_{0}=x_{n}\left[a_{1}+a_{2} x_{n}+\ldots+a_{k} x_{n}^{k-1}\right],
$$

we see that $x_{n}$ divides $p-a_{0}$, and $\left(p-a_{0}\right) / x_{n}$ is $O(n-1)$ invariant. By the argument in the last lemma, $a_{1}$ is $O(n-1)$-invariant. Continuing in this way, we see that each $a_{i}$ is $O(n-1)$-invariant. Now set $\tilde{r}^{2}=x_{1}^{2}+\ldots+x_{n-1}^{2}$; then by Lemma 5.3, each $a_{i}\left(x_{1}, \ldots, x_{n-1}\right)=b_{i}\left(\tilde{r}^{2}\right)=b_{i}\left(r^{2}-x_{n}^{2}\right)$, for some polynomial $b_{i}$. Thus $p=\sum_{i=0}^{k} b_{i}\left(r^{2}-x_{n}^{2}\right) x_{n}^{i}$, i.e., $p$ is a polynomial in $r^{2}$ and $x_{n}$. This completes the proof of Lemma 5.3.

Now let $P_{n}(k)$ denote the homogeneous polynomials of degree $k$ in $\left(x_{1}, \ldots, x_{n}\right)$.

Lemma 5.5. $\operatorname{dim}\left[P_{n^{(k+2)}}^{O(n-1)}\right]=1+\operatorname{dim}\left[P_{n^{(k)}}^{O(n-1)}\right]$.

Proof. Let $p \in P_{n^{(k+2)}}^{O(n-1)}$; then from Lemma 5.4,

$$
p=a_{0} x_{n}^{k+2}+a_{1} x_{n}^{k} r^{2}+a_{2} x_{n}^{k-2} r^{4}+\ldots+a_{k} r^{k+2},
$$

where each $a_{i}$ is a constant; i.e.,

$$
P_{n^{(k+2)}}^{O(n-1)} / r^{2} P_{n^{(k)}}^{O(n-1)}=P_{n^{(k+2)}}^{O(n-1)}=r^{2} P_{n^{(k)}}^{O(n-1)}+\left\langle\operatorname{span} x_{n}^{k+2}\right\rangle .
$$

Thus

$$
p=a_{0} x_{n}^{k+2}+r^{2} q\left(x_{n}, r^{2}\right),
$$

where $q \in P_{n^{(k)}}^{O(n-1)}$; this yields the desired result.

Lemma 5.6. $\Delta: P_{n^{(k+2)}}^{O(n-1)} \rightarrow P_{n^{(k)}}^{O(n-1)}$ is surjective.

Proof. Let $r^{2 b} x_{n}^{k-2(b-1)}, b=0,1, \ldots,\left[\frac{k+2}{2}\right]$, denote an arbitrary basis element of $P_{n^{(k+2)}}^{O(n-1)}(\mathrm{cf}$. Lemma 5.4). Then a computation gives

$$
\begin{aligned}
\Delta\left(r^{2 b} x_{n}^{k-2(b-1)}\right)= & a(a-1) r^{2 b} x_{n}^{k-2 b} \\
& +2 b[n+2(k-b+2)] r^{2 b-2} x_{n}^{k-2(b-1)},
\end{aligned}
$$

where $a=k-2(b-1)$. From this formula, we see that the matrix which represents this linear transformation is of the form

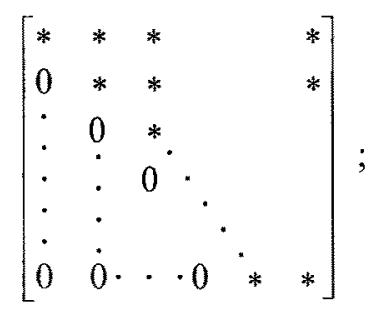

that is, it is upper-triangular, with non-zero entries along the "diagonal." This shows that $\Delta$ is surjective.

From the last lemma, it follows that

$$
\operatorname{dim} \operatorname{ker} \Delta=\operatorname{dim} P_{n^{(k)}}^{O(n-1)}-\operatorname{dim} P_{n^{(k-2)}}^{O(n-1)}=1 .
$$


On the other hand, since $\operatorname{ker}(\Delta)$ is $H_{k}^{O(n-1)}$, we see that $\operatorname{dim} H_{k}^{O(n-1)}=1$, and thus $\operatorname{dim} V_{k}^{o(n-1)}=1$, in view of (5.5). This completes the proof of Proposition 5.2.

Remark. There are subgroups $H$ of $O(n)$, different from $O(n-1)$, whose fixed-point set of the $N^{\text {th }}$-eigenspace of $\Delta$ with respect to $H$ are 1 -dimensional. Thus there bifurcates out of each $u\left(\cdot, p_{k}\right)$ even more solutions than we have described above. We plan to discuss this, and related topics in a future publication.

Acknowledgements. We are grateful to Christoph Pospiech for pointing out an error in a preliminary version, and to Eugene Fabes, Martin Golubitsky, Roger Nussbaum, Paul Rabinowitz, and Joel Spruck for encouraging remarks.

\section{References}

0. Agmon, S.: Lectures on elliptic boundary value problems. Princeton: Van Nostrand 1965

1. Berger, M., Gauduchon, P., Mazet, E.: Le spectre d'une variété Riemannienne. In: Lecture Notes in Mathematics, Vol. 194. Berlin, Heidelberg, New York: Springer 1971

2. Dancer, E.N.: On non-radially symmetric bifurcation. J. Lond. Math. Soc. 20, 287-292 (1979)

3. Gidas, B., Ni, W.M., Nirenberg, L.: Symmetry of positive solutions of nonlinear elliptic equations in $\mathbb{R}^{n}$. Commun. Math. Phys. 68, 202-243 (1979)

4. Helgason, S.: Topics in harmonic analysis on homogeneous spaces. Boston: Birkhäuser 1981

5. Smoller, J., Wasserman, A.: Global bifurcation of steady-state solutions. J. Differ. Equations 39, 269-290 (1981)

6. Smoller, J., Wasserman, A.: Existence, uniqueness, and non-degeneracy of positive solutions of semilinear elliptic equations. Commun. Math. Phys. 95, 129-159 (1984)

7. Smoller, J., Wasserman, A.: Symmetry-breaking for positive solutions of semilinear elliptic equations. Arch. Ration. Mech. Anal. (to appear)

8. Smoller, J., Wasserman, A.: An existence theorem for positive solutions of semilinear elliptic equations. Arch. Ration. Mech. Anal. (to appear)

9. Smoller, J.: Shock waves and reaction-diffusion equations. Berlin, Heidelberg, New York, Tokyo: Springer 1983

10. Vanderbauwhede, A.: Local bifurcation and symmetry. Research Notes in Mathematics, No. 75. Boston: Pitman 1982

Communicated by L. Nirenberg

Received November 7, 1984; in revised form March 10, 1986

Note added in proof. The application of Theorem 2.2 is not quite correct since $H^{1}$ functions need not have normal derivatives on $S^{n-1}$; we thank Christoph Pospiech for pointing this out to us. We remedy this by noting that the existence of infinitesimal symmetry-breaking, Theorem 4.6 , implies that the Conley index of the rest point $u(\cdot, p)$ of the parabolic equation $u_{i}=\Delta u+f(u)$ changes as $p$ goes from $p_{k}$ to $p_{k+1}$.

This means that actual bifurcation must occur (see [10, Chap. 22]). 